\title{
Pulmonary endometriosis: Clinico-pathological approach to diagnostics and treatment
}

\author{
Dvorakovskaya IV', Pechenikova VA², Ariel BM ${ }^{3,6 *}$, Platonova IS', Orzheshkovskiy OV ${ }^{4,5}$, Pichurov AA ${ }^{5,6}$ and Yablonskiy PK ${ }^{1,4,6}$ \\ ${ }^{1}$ Institute for Pulmonologic Research, Academician I.P.Pavlov's St Petersburg State First Medical University, Healthcare Ministry of Russian Federation, Russia \\ ${ }^{2}$ I.I.Mechnikov's State North-West Medical University, Healthcare Ministry of Russian Federation, Russia \\ ${ }^{3}$ City of St Petersburg State Bureau for Pathology, Russia \\ ${ }^{4}$ St Petersburg State University, Russia \\ ${ }^{5}$ State Multidisciplinary Hospital No.2, St Petersburg, Russia \\ ${ }^{6}$ St Petersburg Federal Research Institute of Phthisiology and Pulmonology, Healthcare Ministry of Russian Federation, Russia
}

\begin{abstract}
Background: A pulmonary endometriosis is one of the relatively rare forms of the extragenital endometriosis and in accordance to its clinico-pathological symptoms is considered within the terms of the "thoracic endometriosis syndrome", i.e. when functioning endometrium is found in the pleura, diaphragm, viae respiratoriae or the pulmonary parenchyma itself.

Objectives: 42 cases of this disease were observed. The patients age ranged from 16 to 61 years, thus the average being $40.5 \pm 0.4$ years.

Results: In the majority of cases the endometriosis affected the diaphragm and was accompanied by a pneumothorax with the corresponding symptoms complex, while pulmonary foci occurred rarer, being asymptomatic or feigning some surgical and other pathology. The surgery and the histological study were crucial in verifying the diagnosis. Progesterone and estrogen receptors, and $\mathrm{Ki}-67$ production were detected immuno-histochemically in the endometrioid tissue of the lung foci.
\end{abstract}

Conclusion: The use of the comprehensive histological and immuno-histochemical methods was required to make the final diagnosis of the pulmonary endometriosis more reliable. It has a great practical significance being one of the most important pre-requisites for prescription of an adequate treatment.

\section{Introduction}

Endometriosis is a growth of the tissue similar to the endometrium outside the uterine cavity which is accompanied by a chronic inflammatory reaction, leading in most cases to the development of a pain syndrome and/or infertility. Currently, it is one of the most common gynecological diseases. This pathology occurs in $2-10 \%$ of women of reproductive age and almost in $50 \%$ of women with infertility $[1,2]$.

One of the relatively rare forms of the endometriosis is characterized by the development of the endometrial tissue outside the reproductive system. Extragenital foci may exist independently both as separate formations and as components of the concomitant lesions $[3,4]$. The cases of the extragenital endometriosis make $6-8 \%$ of the total number of the endometriosis observed. Among the organs unrelated to the reproductive system, there are the intestines, the urinary tract, and the lungs that are most frequently affected [5].

Hart was the first to diagnose the pulmonary endometriosis (PE) posthumously in 1912. He observed in a woman, aged 72, numerous subpleural nodes varying from the size of a pea to that of a walnut. The histological examination showed adenomyosis of the uterine origin; a tumor in the patient's uterus having been removed 22 years prior to that [6].

Over the last decade the number of publications on the endometriosis has markedly increased. The concept of the "thoracic endometriosis syndrome" (TES) has been developed and used in cases when the functioning endometrium is found in the pleura, the diaphragm, the viae respiratoriae or the pulmonary parenchyma itself $[7,8]$.

Channabasavaiah and Joseph [9] have presented results of an analysis of 110 clinical cases of the histologically verified intrathoracic endometriosis that were published between 2001 and 2007. Legras et al. [10] have studied retrospectively 229 cases of the pneumothorax in women described in the specialist literature written in English from 2000 to 2011 and state that the TES being diagnosed in 54 cases (24 per cent).

The question of how a functioning endometrium gets into the chest remains as yet open $[6,11,12]$. Sampson [13] was the first to suggest that the endometrium is transferred from the uterine cavity into the abdominal one through the fallopian tubes by way of the so-called "retrograde menstruation", that is, the discharge of some amount of

Correspondence to: Boris M. Ariel Saint-Petersburg Federal Research Institute of Phthisiopulmonology, Healthcare Ministry of Russian Federation; Ligovsky prospekt 2/4 193036 Saint-Petersburg Russia, Tel: (812) 534-3791; E-mail: arielboris@rambler.ru

Key words: thoracic endometriosis syndrome, extragenital endometriosis, pulmonary endometriosis, diaphragm lesion, pneumothorax, histological verification of diagnosis

Received: November 28, 2017; Accepted: December 18, 2017; Published: December 22, 2017 
blood into the pelvic cavity during menstruation. This kind of reflux occurs in a large number of healthy women [14]. Sampson [13] also hypothesized an existence of the metastatic, or embolic endometriosis, which allows one to speculate about hematogenous routes of the endometriosis propagation. Hobbs and Bortnick [11] confirmed this hypothesis experimentally introducing the endometrium suspension into the ear veins of rabbits, which resulted in the development of the $\mathrm{PE}$ in 79 per cent of cases.

The TES manifests itself most often in the recurrent pneumothorax, haemoptysis, cough or chest pain, and more rarely in the haemothorax associated with the menstrual $[2,7,15-17]$. The $\mathrm{PE}$ is one of the rarest forms of the TES. The most important clinical manifestations of the former are cough, haemoptysis, and a feeling of heaviness in the chest (a «heavy fur coat» symptom) that occur during menses, but not necessarily in every menstrual cycle.

According to the literature, pneumothorax associated with the TES is found in approximately 10-24 per cent of women with a spontaneous pneumothorax $[7,16]$.

X-rays chest examination (mainly HRCT) reveals four variants of the pathology: linear, or reticular opacities, small nodules, cystic airspaces, thickened alveolar septa. These radiological findings, particularly those combined with a haemoptysis and chest pain are often regarded as an evidence of a tuberculous or a neoplastic nature of the pulmonary lesion.

A diaphragm lesion is usually detected by accident during a surgical intervention due to pneumothorax. Prior to the surgery this pathology is very seldom detected because of a limited extent of the lesions and low resolution of radiological methods.

Almost without exception, the disease affects a tendinous centre of the right diaphragm dome and may appear in two kinds. The first one takes the form of blue or purple endometrious implants under the parietal pleura up to $5 \mathrm{~mm}$ in size. The second are round or slit-like defects in the diaphragm.

The lack of specific symptoms, of laboratory markers, and characteristic radiological patterns makes the preoperative diagnosis of PE difficult. It is often finally diagnosed only after a confirmation by the histological methods, among them the immuno-histochemistry, including estrogen and progesterone receptors expression [15].

Therefore, the surgical intervention is often necessary on diagnostic and therapeutic purposes. Though the operation does verify the diagnosis as a rule and removes any heterotopic endometrium lesions, this kind of treatment remains incomplete [7, 17] without a suppressive hormonal therapy. It aims at an ovulation blocking, a suppression of the estrogen secretion and epithelial growth supression in the endometrious focus [18].

A relatively small number of the TES cases described in the world specialist literature and the lack of its pathognomonic clinical picture prompted us to summarize our own experience in the diagnostics and treatment of this disease.

\section{Methods}

We've analyzed 42 cases of the TES observed in our hospitals over the period from 2004 to 2016 . The patients age ranged from 16 to 61 years, thus the average being $40.5 \pm 0.4$ years.

35 patients were hospitalized due to a spontaneous pneumothorax, 3 with rounded tumorous masses, 1 with a cyst, and 3 with an infiltration in the lung tissue. Significantly, in 41 patients the pathological process was localized in the right lung.

\section{Results}

The main complaints of all hospitalized patients are listed in the Table 1 .

In most cases, there was a chest pain (85.7 per cent); haemoptysis was found less often (9.5 per cent). Dyspnea was noted only in 31 patients with pneumothorax; 6 women suffered from infertility. In the anamnesis, 5 patients had uterine myoma, and there was ovary endometrial cyst in 2 patients.

HRCT of the chest detected rounded shadows in the lungs (3 cases), infiltrates with thin-walled cavities ( 6 cases), lung cyst (1 case), bullae of various diameter ( 6 cases), and a mass in the paravertebral region considered as a neurinoma (1 case) (Figure 1). In the same time no changes in the lung tissue were detected in 26 of 42 cases (61.9 per cent of the patients).

Unfortunately, a preoperational examination doesn't allow us to suggest the TES in any case, as CA-125 expression doesn't indicate the possibility of it either. At the same time, a retrospective analysis of the post-operative data suggested that an essential prerequisite to this diagnosis did exist.

Pre-operational diagnoses were the following: primary spontaneous pneumothorax in 35 cases, infiltrative pulmonary tuberculosis in 3 cases, lung haemosiderosis, neurinoma of mediastinum, lung tumour, and lung cyst - each being in one.

The surgical intervention was performed in 37 of 42 patients. Of those 35 women with pneumothorax, 30 ( 85.7 per cent) were operated. Atypical lung resection was performed in 27 cases $(90$ per cent of the patients), costal pleurectomy in 28 cases (93.3 per cent) and the resection of the diaphragm tendon centre in 25 cases (83.3 per cent).

Of 7 patients without pneumothorax 6 were subjected to the atypical lung resection and 1 to the lobectomy.

In all cases a histological examination of the surgical material was performed. Paraffin sections were stained with haematoxylin and eosin, alcian blue, and picrofuchsin by van Gieson. Immunohistochemistry was performed using antibodies to progesterone and estrogen receptors as well as mouse monoclonal antibodies to Ki-67 and bcl 2 (Dako, Denmark).

In correlating clinical and histological data, the TES was diagnosed in all cases and may be subdivided into following categories: perforation of the right dome of the diaphragm with histologically identfied endometrial implants (6 patients); intrapulmonary endometrial heterotopias in the form of nodes, infiltrates and cysts (11 patients); multifocal lung and diaphragm lesions (1 patient). A perforation of the diaphragm right dome without histologically identified endometrium ("porous diaphragm") was observed in 24 cases.

Table 1. Complaints of patients with the thoracic endometriosis syndrome.

\begin{tabular}{|c|c|c|}
\hline \multirow{2}{*}{ Complaints } & \multicolumn{2}{|c|}{ Patients (n=42) } \\
\cline { 2 - 3 } & abs. number & per cent \\
\hline Chest pain & 36 & 85,7 \\
\hline Dyspnea & 31 & 73,8 \\
\hline Dry cough & 21 & 50,0 \\
\hline Infertility & 6 & 14,3 \\
\hline Haemoptysis & 4 & 9,5 \\
\hline
\end{tabular}




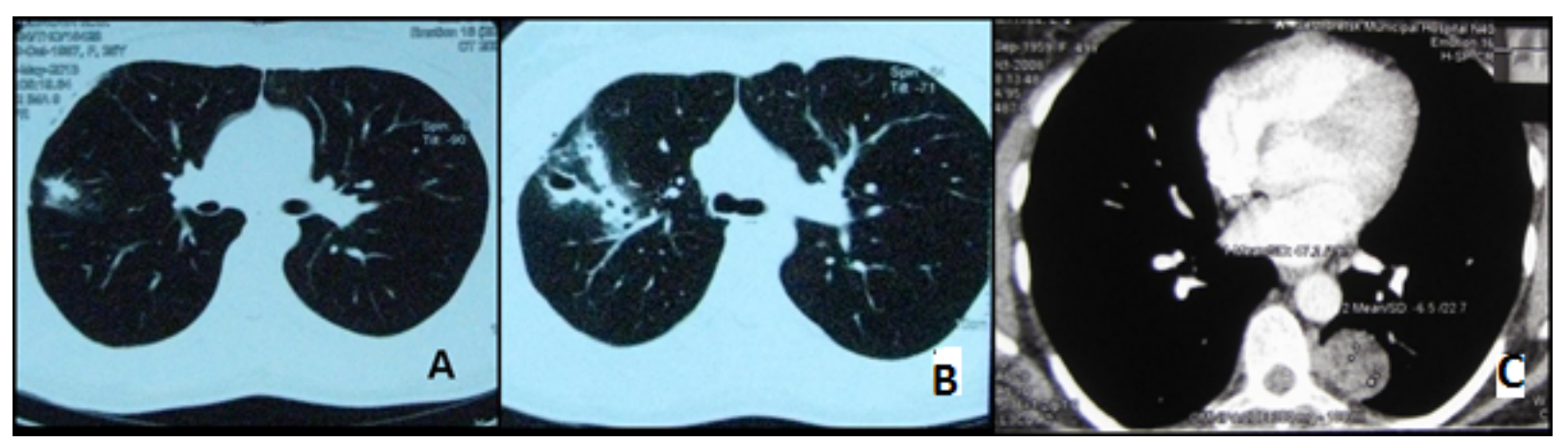

Figure 1. HRCT chest scans of patients with pulmonary endometriosis A - lung tissue infiltration; B -small cavities; C - peripheral nodules.

The macroscopic assessment of removed lungs detected cysts with dark brown material ( 6 cases). The walls of the cysts were thick, their inner surface smooth ( 5 cases), or with papillary outgrowths (1 case). 5 remaining observations revealed small hemorrhage foci in the subpleural areas.

The microscopic examination detected numerous clusters of endometrial glands with a cytogenic stroma. The structure of these glands corresponded to various phases of the menstrual cycle 9 (viz. a proliferation or secretion stage) presented in different quantities. The endometrial type of epithelium of the glands was usually pseudostratified, flattened or columnar; cell nuclei were located at the different levels; the cytoplasm contained secret drops; here and there some signs of epithelium desquamation were noted. In the alveolar lumina and the lung interstitium hemosiderin deposits were often found. The glandular stroma was represented by elongated cells with spindle-shaped nuclei, in some places with decidualization features (Figure 2). In 5 cases a cystic transformation of glands was observed along with haemorrhages around them. Some haemosiderin deposits and a fibroblastic transformation of a cytogenic stroma were evident in the most cases.

In all cases the immuno-histochemical study was performed. It revealed the progesterone and estrogen receptors expression in the gland epithelium and cytogenic stroma cells (Figure $3 \mathrm{~A}, \mathrm{~B}$ ) as well as expression of the oncoprotein bcl-2 in the gland epithelium and moderate proliferative activity (Ki-67) in the cytogenic stroma (Figure $3 \mathrm{C}$ ).

Shown below are two examples from our observations.

Example 1. Patient F., 52 years old. The clinical diagnosis was a tumor of the right lung lower lobe. Six years before, extirpation of the uterus without appendages was performed on the account of the uterus myoma with adenomyosis; the patient suffered from infertility. 6 years after the surgery there were complaints about periodic aching pains in the chest, cough and haemoptysis. The radiography of the thorax revealed a round shadow of $4 \mathrm{~cm}$ in diameter in the lower lobe of the right lung. HRCT showed a rounded formation, $4 \times 3 \times 2,5$ $\mathrm{cm}$ in size, heterogeneously structured with well-defined contours. The examination failed to verify the precise diagnosis, so the right lower lobectomy was performed. Macroscopically, a "honeycomb" type formation with well-defined contours and small cavities up to $0.7 \mathrm{~cm}$ filled with thick brown liquid was detected. There was a dense homogeneous fibrous tissue between cavities. The histological examination revealed cystic transformed endometrioid glands with a proliferating epithelium on their walls as well as a papillae formation and a fibrous cytogenic stroma, along with a leiomyomatous hyperplasia around glandular structures (Figure 4).

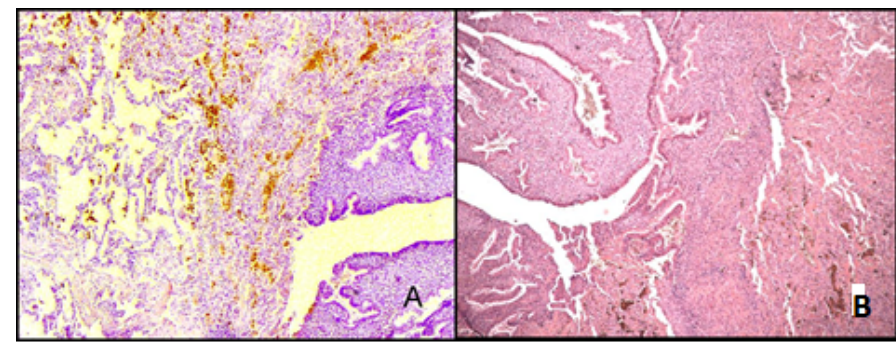

Figure 2. Pulmonary endometriosis. A - islets of endometrial glands proliferation. In the alveolar lumina there are hemosiderin deposits. Haematoxylin and eosin staining (X 100); B - proliferating epithelium of the glands with secret drops in cytoplasm. Stroma with decidualization features. Haematoxylin and eosin staining (X 160).

Example 2: Patient A., 42 years old. Clinical diagnosis was the rightside spontaneous pneumothorax. On the first day of the menstrual cycle pain in the right half of the chest appeared. It was accompanied by a dry cough and a slight dyspnea. Chest radiography revealed a right-side pneumothorax with a 50 per cent atelectasis and a shift of the mediastinum to the left. Upon admission to the hospital a right-side diagnostic thoracoscopy was performed with a drainage of the pleural cavity. The tendon center of the diaphragm showed perforations and endometrial implants (Figure 5). HRCT of the chest revealed no changes in the lung tissue. The right-side videothoracoscopy, resection of the diaphragm tendon center and subtotal costal pleurectomy were performed. Macroscopically, some perforations in the tendon centre and brownish nodules with well-defined contours up to $0.5 \mathrm{~cm}$ were detected. They were located on the pleural diaphragm surface without penetrating through it. The histological examination showed some cystic endometriod lesions on the fibrous background.

In this case, as well as in all others, the onset and the disappearance of clinical and radiological symptoms coincided with the beginning and the end of the menstrual cycle. Nevertheless, this fact escaped the physisians.

\section{Discussion}

As a clear proof of the words once uttered by the well-known Russian oncomorphologist Golovin $[19,20]$, the extragenital endometriosis confirms that every tissue is a historically formed stable unity possessing certain properties and morphogenetic potencies, which do not disappear completely even in case of abnormal interrelations between an organism and a tumor. This approach is extremely important for understanding the $\mathrm{PE}$ as a pathological process toto coelo.

In most of our observations, the endometriosis affected the diaphragm and was accompanied by the pneumothorax with a corresponding symptoms complex, while the pulmonary heterotopia 


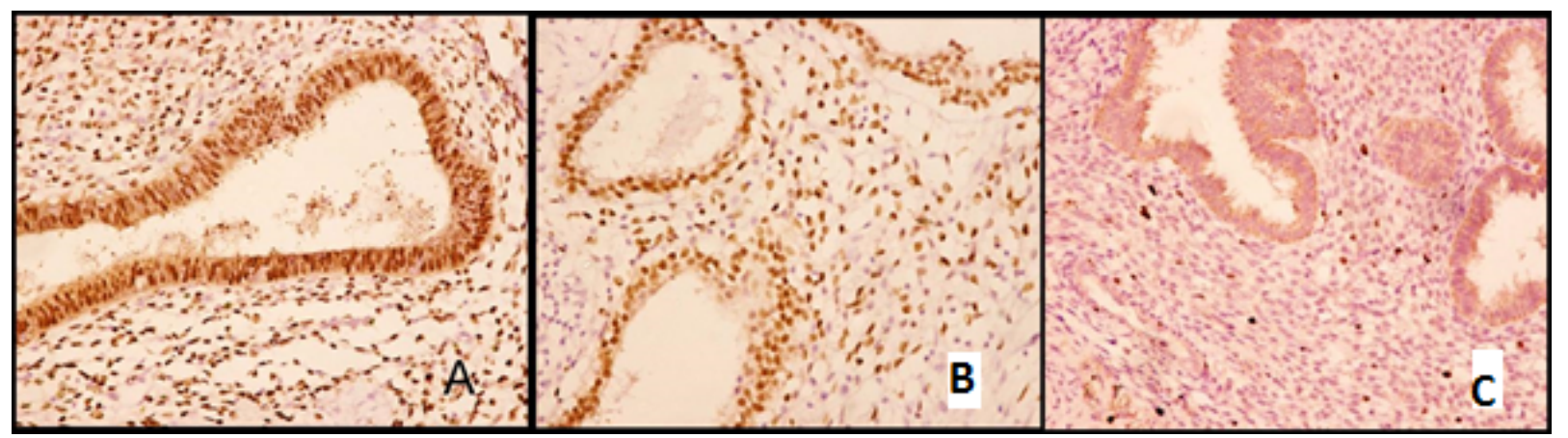

Figure 3. Pulmonary endometriosis: A - expression to progesterone receptors in glandular epithelial cells (X200); B - identical with estrogen receptors (X200); C - Ki-67 expression in the cytogenic stroma (X100).

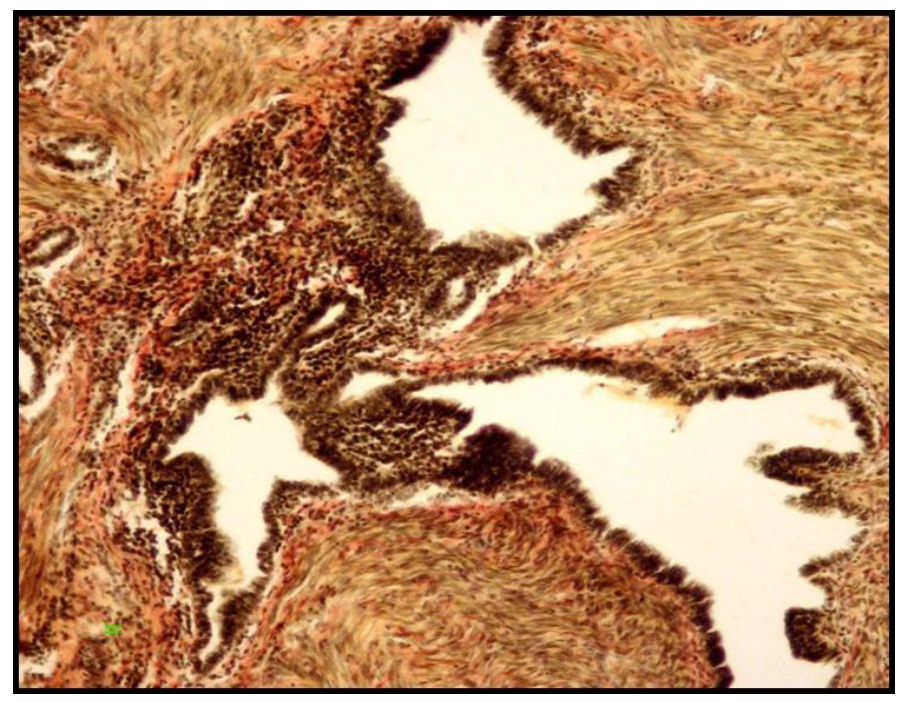

Figure 4. Pulmonary endometriosis with papillary structures and leiomyomatous hyperplasia. Van Gieson staining (X 160).

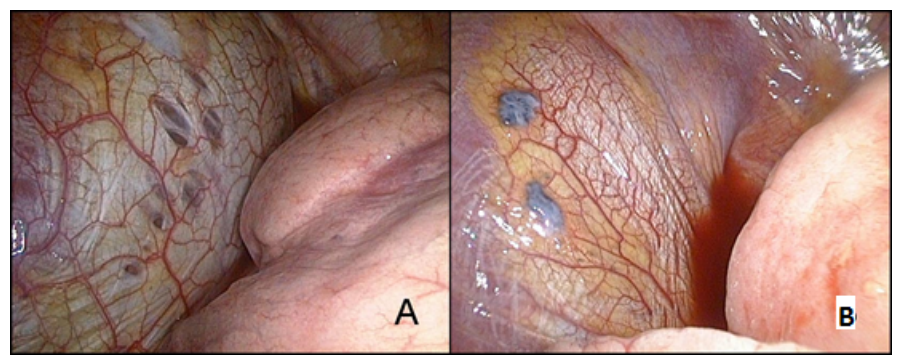

Figure 5. Diaphragm lesions revealed in operation. A - perforations in the diaphragm tendon center; B - endometrioid implants.

occurred rarer being asymptomatic or feigning certain surgical and other pathology.

It is worth mentioning that the lesion of the left half of the chest was diagnosed only in one case (viz. implants in the left dome of the diaphragm and the left lung), which confirms the fact that TES is the right-sided pathology. We suggest that the reason of this selectivity is the fact that the right dome of the diaphragm together with the liver, although not rigidly bound, works during the breathing like a piston providing a "suction effect". Supposedly, this is also the reason why it is exactly the space under the right diaphragm dome where certain amount of the menstrual blood can get from the pelvis. The blood resorption is carried out with the active participation of the lymphatic apparatus of the peritoneum [21]. Endometrial fragments reach the subpleural space through the lymphatic vessels perforating the diaphragm and remain fixed there in most cases. The subsequent necrosis and lysis of the endometrium may lead to the perforation defects. Hypothetically it is possible that in rare cases small endometrium fragments reach the superior vena cava through the mediastinum lymphatic vessels and then penetrate into the pulmonary parenchyma forming nodes, or cysts.

The clinical and morphological hypodiagnostics of the TES is conditioned not only by its rarity, but by the lack of the comprehensive complex analysis and correlation of the anamnestic, clinical, and histological data as well.

Given the increasing incidence of the endometriosis, the pulmonary one among others, it seems essential to include this disease into the differential diagnostics range, particularly where women of childbearing age with the unclear right-sided pulmonary pathology and compromised gynecological history are involved. In such cases, it is advisable to recruit gynecologists so as to exclude the adenomyosis and the pelvic endometriosis. In the case of the obscure recurrent pulmonary diseases it is imperative to carry out the MRI of the small pelvis organs.

Our data allow to determine some important and reliable features of the TES as follows: the predominantly right-side localization of the disease, the relatively young age of the patients, the cyclic recurrence of the haemoptysis and discomfort or pain in the chest, the recurrent right-side pneumothorax, the correlation of the onset and/ or regression of the radiological symptoms with the beginning and/or end of the menstrual cycle, one or the other of gynecological problems (viz. infertility, pelvic pain, algomenorrhea), and surgeries on the pelvic organs in the anamnesis.

The diagnostic surgery requires an extensive use of the whole operation material with cutting out samples for the histological examination of all affected lung areas, since the endometriosis microscopic features in different, even neighbouring parts of the lung may vary considerably. From the diagnostic standpoint, the essential morphological distinctive feature of the PE is a combination of the stability, progression or regression signs within the same or in the adjacent samples of the lung tissue. To make the final diagnosis of the TES more reliable, the use of the comprehensive histological and immuno-histochemical methods is required. The precise diagnosis of the endometriosis is of great practical importance because it is a prerequisite for a selection of the adequate treatment.

\section{References}

1. Tsvelev YV, Abashin VG, et al. (2007) Endometriosis: modern views on the etiology, terminology and classification. Vestnik Rus Military Medical Academy 4: $42-47$ [In Russian]. 
2. Olive DL, Schwartz LB (1993) Endometriosis. $N$ Engl J Med 328: 1759-1769. [Crossref]

3. Ozhiganova IN (2009) Endometriosis and endometrioid disease (working standards of postmortem examination). $\mathrm{SPb}$. [In Russian].

4. Harada M, Osuga Y, Izumi G, Takamura M, Takemura Y, et al. (2011) Dienogest, a new conservative strategy for extragenital endometriosis: a pilot study. Gynecol Endocrinol 27: 717-720. [Crossref]

5. Bergqvist A (1993) Different types of extragenital endometriosis: a review. Gynecol Endocrinol 7: 207-221. [Crossref]

6. Joseph J, Sahn SA (1996) Thoracic endometriosis syndrome: new observations from an analysis of 110 cases. Am J Med 100: 164-170. [Crossref]

7. Alifano M, Trisolini R, Cancellieri A, Regnard JF (2006) Thoracic endometriosis: current knowledge. Ann Thorac Surg 81: 761-769. [Crossref]

8. Flieder DB, Moran CA, Travis WD, Koss MN, Mark EJ (1998) Pleuro-pulmonary endometriosis and pulmonary ectopic deciduosis: a clinicopathologic and immunohistochemical study of 10 cases with emphasis on diagnostic pitfalls. Hum Pathol 29: 1495-1503. [Crossref]

9. Channabasavaiah AD and Joseph JV (2010) Thoracic endometriosis: revisiting the association between clinical presentation and thoracic pathology based on thoracoscopic findings in 11 patients. Medone. Baltimore 89: 183-188. [Crossref]

10. Legras A, Mansuet-Lupo A, Rousset-Jablonski C, Bobbio A, Magdeleinat P, et al. (2014) Pneumothorax in women of child-bearing age: an update classification based on clinical and pathologic findings. Chest 145: 354-360.11. [Crossref]
11. Hobbs JE and Bortnick AR. (1940) Endometriosis of the lung; an experimental and clinical study. Am J Obstet Gynecol 40: 832-843.

12. Pichurov AA, OrzheshkovskiÄ OV, Dvorakovskaia IV, Romanova LA, Ivanishchak $\mathrm{BE}$, et al. (2014) [Thoracic endometriosis--the rare pathology in thoracic surgery] Vestn Khir Im I I Grek 173: 26-29. [Crossref]

13. Sampson JA (1927) Peritoneal endometriosis due to menstrual dissemination of endometrial tissue into the peritoneal cavity. Am J Obstet. Gynecol 14: 422-469.

14. Adamyan LV, Kulakov VI, and Andreeva EN (2006). Endometriosis. M., Medizina. [In Russian].

15. Agarwal N. and Subramanian AA (2010) Endometriosis. Morphology, clinica presentations, and molecular pathology. $J$ Lab Physicians 10: 1-9. [Crossref]

16. IablonskiÄ PK, Pichurov AA, OrzheshkovskiÄ OV, Petrun'kin AM, Goncharuk IV (2014) [Features of spontaneous pneumothorax in female]. Vestn Khir Im I I Grek 173: 89-95. [Crossref]

17. Terada Y, Chen F, Shoji T, Itoh H, Wada H, et al. (1999) A case of endobronchial endometriosis treated by subsegmentectomy. Chest 115: 1475-1478.18. Koizumi T, Inagaki H, et al. (1999) Successful use of gonadotropin-releasing hormone agonist in a patient with pulmonary endometriosis. Respiration 66: 544-546. [Crossref]

18. Golovin DI (1975) Atlas of human tumors. Leningrad, Medizina. [In Russian].

19. Abu-Hijleh MF, Habbal OA, Moqattash ST (1995) The role of the diaphragm in lymphatic absorption from the peritoneal cavity. J Anat 186: 453-467. [Crossref]

Copyright: (C2017 Dvorakovskaya IV. This is an open-access article distributed under the terms of the Creative Commons Attribution License, which permits unrestricted use, distribution, and reproduction in any medium, provided the original author and source are credited. 